\title{
Densitometria da vértebra dorsal, osso pleural e osso neural em tartarugas verdes hígidas por tomografia computadorizada quantitativa
}

\author{
Densitometry of dorsal vertebrae, pleural bone and neural bone in healthy green sea turtles by \\ quantitative computed tomography
}

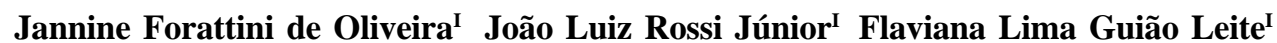 \\ Daniel Capucho de Oliveira' ${ }^{\mathrm{II}}$ Lorena Adão Vescovi Séllos Costa ${ }^{\mathrm{III}}$ Ieverton Cleiton Correia da Silva ${ }^{\mathrm{III}}$ \\ Marcelo Weinstein Teixeira ${ }^{\mathrm{III}}$ Fabiano Séllos Costa ${ }^{\mathrm{III}^{*}}$
}

\section{RESUMO}

A tomografia computadorizada quantitativa (TCQ) é uma técnica com alta sensibilidade, aplicabilidade e precisão no diagnóstico de perda de massa óssea, entretanto a falta de valores de normalidade para animais silvestres limita a sua aplicação clínica. O presente estudo objetiva estabelecer valores médios de radiodensidade do osso trabecular das vértebras dorsais, osso pleural e osso neural de tartarugas marinhas juvenis hígidas utilizando a TCQ. Para isso, utilizouse um grupo experimental de cinco animais que foram submetidos a exames de tomografia computadorizada helicoidal, sendo as mensurações da radiodensidade óssea realizadas ao nível da terceira, quarta e quinta vértebras dorsais. Os valores médios de radiodensidade do osso trabecular das vértebras dorsais, osso pleural e osso neural foram,

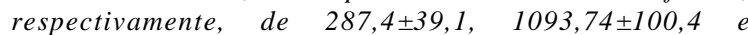
1089,6 $\pm 86,5$ unidades Hounsfield (HU). Estudos complementares devem ser realizados em diferentes espécies e faixas etárias de quelônios para favorecer a aplicação clínica desta técnica.

Palavras-chave: quelônios, coluna, densidade mineral óssea, tomografia computadorizada quantitativa, Chelonia mydas.

\section{ABSTRACT}

Quantitative computed tomography (QCT) is a technique with high sensitivity, applicability and accuracy in the diagnosis of bone loss; however the lack of normal values for wild animals limits their clinical application. This study aims to establish medium values radiodensity trabecular bone of the dorsal vertebrae, bone pleural and neural bone in healthy juvenile sea turtles using the QTC. For this we used an experimental group of five animals that underwent helical computed tomography, and measurements of bone radiodensity conducted at the level of the third, fourth and fifth dorsal vertebrae. The medium values of radiodensity trabecular bone of the dorsal vertebrae, pleural bone, and neural bone were respectively $287.4 \pm 39.1,1093.74 \pm 100.4$, and $1089.6 \pm 86.5$ Hounsfield units (HU). Further studies should be performed in different species and ages of turtles to encourage the clinical application of this technique.

Key words: chelonians, spine, bone mineral density, quantitative computed tomography, Chelonia mydas.

\section{INTRODUÇÃO}

A atividade exploratória humana causa diminuição não intencional da população de tartarugas marinhas (LUTCAVAGE et al., 1996). Um maior conhecimento dos aspectos anatômicos, fisiológicos e das enfermidades que acometem as tartarugas marinhas é importante para a sua conservação, portanto técnicas diversas de diagnóstico por imagem contribuem significativamente para o manejo clínico e cirúrgico de tartarugas marinhas (SILVERMAN, 2006).

Atualmente, existem diversos métodos de diagnóstico por imagem que podem ser empregadas para mensuração da densidade mineral óssea (DMO). As mais utilizadas são a ultrassonografia quantitativa (LAUER et al., 2009), a tomografia computadorizada quantitativa (TCQ) (CHALMERS et al., 2006, SAEED

IDepartamento de Medicina Veterinária, Centro Universitário Vila Velha (UVV), Vila Velha, ES, Brasil.

IIDepartamento de Medicina Veterinária, Universidade Federal do Paraná (UFPR), Curitiba, PR, Brasil.

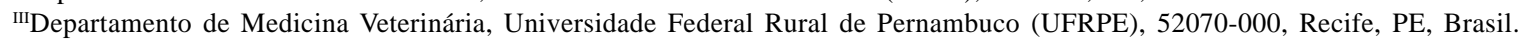
E-mail: fabianosellos@hotmail.com.*Autor para correspondência. 
et al., 2009) e a absorciometria por dupla emissão de raios-X (DEXA) (QUARLES, 1992). Dentre essas técnicas citadas, a TCQ e a DEXA são as mais precisas e menos invasivas (MARIRIC \& GLUCK, 2004; ADAMS, 2009). Porém, a TCQ é considerada um exame mais sensível e fornece informações sobre a morfometria óssea, das quais podem ser extraídos parâmetros biomecânicos (MARICIC \& GLUCK, 2004; ADAMS, 2009).

Alterações no metabolismo mineral ósseo em tartarugas marinhas são frequentemente descritas em animais de vida livre e em animais que vivem em cativeiro (GEORGE, 1996; MADER, 2006). A maior limitação para a aplicação da TCQ na medicina veterinária das diversas espécies de tartarugas marinhas está relacionada à falta de valores de normalidade, impossibilitando uma avaliação comparativa. O presente estudo objetiva observar os valores médios de radiodensidade do osso trabecular das vértebras dorsais, osso pleural e osso neural de tartarugas marinhas juvenis hígidas utilizando a técnica de TCQ.

\section{MATERIAL E MÉTODOS}

Para a realização deste estudo foram utilizadas cinco tartarugas verdes Chelonia mydas juvenis hígidas. Os animais foram capturados na mesma data no efluente final do ArcelorMittal (Companhia Siderúrgica de Tubarão), Espírito Santo, Brasil. O método de captura foi por tarrafa, sendo posteriormente as tartarugas encaminhadas para exame clínico e realização dos exames tomográficos. Nenhuma das tartarugas apresentava maturidade sexual, não sendo possível a determinação sexual.

Os animais apresentavam-se em bom estado nutricional, sem alterações ao exame clínico e com peso de $6,8 \pm 0,7 \mathrm{~kg}(5,7-7,3 \mathrm{~kg})$. As dimensões do casco foram determinadas, obtendo-se, em média, 41,0 1 ,6cm (38,5$42,3 \mathrm{~cm})$ de largura e $35,0 \pm 1,6 \mathrm{~cm}(34,0-36,8 \mathrm{~cm})$ de comprimento. Posteriormente à realização dos exames de tomografia computadorizada (TC) e ao término dos efeitos promovidos pelos fármacos anestésicos, os animais foram soltos na praia de Camburi, Vitória, Espírito Santo, Brasil.

Para a contenção química dos animais na hora do exame de TC, realizou-se a administração intravenosa de propofol na dose de $5 \mathrm{mg} \mathrm{kg}^{-1}$. Os animais foram posicionados em decúbito ventral, sendo realizados os exames de TC com o aparelho GE HiSpeed FXI e protocolo com $140 \mathrm{kVp}$ e auto mA na velocidade de uma rotação por segundo. A aquisição das imagens foi obtida em cortes transversais de 3mm de espessura com filtro para tecido ósseo.
Antecedendo os exames, o aparelho foi calibrado para melhor padronização dos resultados. Não foi realizada a administração de contraste radiográfico por via intravenosa ou oral. Após o exame tomográfico e digitalização das imagens, confeccionaram-se imagens de cortes axiais dos ossos pleurais, ossos neurais e corpos vertebrais (Figura 1A) ao nível da segunda (Figura 1B), terceira e quarta vértebras dorsais.

Para estimativa dos valores individuais de radiodensidade óssea de cada animal do grupo experimental, calculou-se o valor de atenuação em unidades Hounsfield (HU) dos ossos neurais, pleurais (direito e esquerdo) e do corpo vertebral a partir da média das regiões de interesse analisadas ("ROIs regions of interest”). Cada ROI apresentava uma área de $1,0 \pm 0,1 \mathrm{~mm}^{2}$ para avaliação densitométrica do osso trabecular do corpo vertebral e de $0,3 \pm 0,1 \mathrm{~mm}^{2}$ para avaliação da radiodensidade dos ossos pleurais e neurais, sendo gerado automaticamente um valor de radiodensidade óssea da região selecionada. A partir dos dados obtidos, realizou-se análise estatística descritiva da radiodensidade das regiões de interesse, obtendo-se os valores de média e desvio padrão. A comparação dos valores de radiodensidade óssea entre os diferentes níveis de corte utilizados para a avaliação densitométrica foi realizada por meio do Teste $t$ de student, com o nível de significância 0,05.

\section{RESULTADO E DISCUSSÃO}

Descrições anatômicas sobre as estruturas ósseas da coluna vertebral de tartarugas marinhas são citadas na literatura. O esqueleto axial é composto de carapaça, osso plastrão, vértebras, costelas e derivações das costelas (VALENTE et al., 2007). A carapaça é formada por escudos que formam a camada mais externa do casco e pelas placas ósseas que são os principais componentes estruturais do casco. Os múltiplos escudos se sobrepõem às placas ósseas (ossos neurais, pleurais e periféricos), que são fundidos com as costelas ou vértebras formando uma estrutura única. Os ossos neurais são aqueles que recobrem a coluna vertebral. Os ossos pleurais são formados pelas costelas e pelas extensões dermais ossificadas, enquanto que os ossos periféricos formam as margens da carapaça (ABOUMADI et al., 2004; VALENTE et al., 2007).

A TC é um método não-invasivo de diagnóstico por imagem que proporciona significativas vantagens para a detecção de enfermidades, sendo considerada uma técnica ideal para o diagnóstico de afecções esqueléticas e de tecidos moles em quelônios (GUMPENBERGER \& HENNINGER, 2001). Apesar disso, informações referentes aos achados 


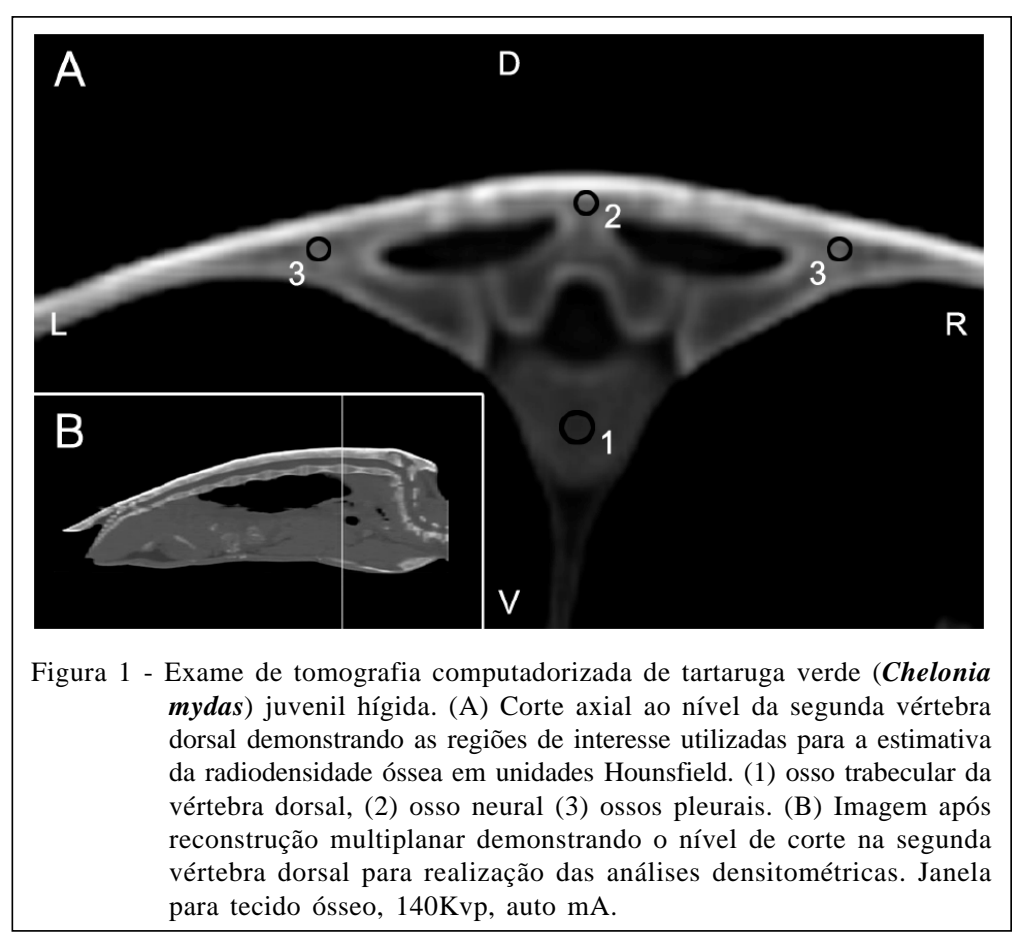

tomográficos de tartarugas marinhas são pouco descritas na literatura veterinária. Em comparação com a radiografia convencional, a TC proporciona melhor distinção das densidades específicas dos tecidos, assim como permite o diagnóstico de discretas alterações no tamanho, formato, contorno e posicionamento de diversos órgãos (GAUDRON et al., 2001). VALENTE et al. (2007) forneceram grande contribuição para um melhor detalhamento da anatomia tomográfica de tartarugas marinhas após realização de exames de TC de tartarugas marinhas cabeçudas (Caretta caretta) juvenis, favorecendo a identificação dos ossos da coluna vertebral e dos órgãos clinicamente importantes presentes na cavidade celomática. Nenhum estudo foi encontrado na literatura consultada citando valores densitométricos dos ossos que compõem a carapaça de tartarugas marinhas.

Diversas alterações do metabolismo mineral ósseo podem acometer répteis, já sendo descrito casos de hiperparatireoidismo secundário renal, hiperparatireoidismo secundário nutricional, osteopatia hipertrófica, osteomalácia, osteopetrosis e doença de Paget (MADER, 2006). Fatores nutricionais são associados à desmineralização óssea, sendo as causas mais comuns relacionadas à deficiência de cálcio e fósforo na alimentação e desequilíbrio na relação cálciofósforo. Tartarugas marinhas que vivem em cativeiro são altamente propensas a desenvolver alterações ósseas, uma vez que sua alimentação quando controlada por humanos pode gerar problemas nutricionais (GEORGE, 1996; MADER, 2006).

Apesar das técnicas de diagnóstico por imagens serem pouco utilizadas na avaliação semiológica de quelônios, esses exames complementares podem contribuir significativamente no manejo desses animais (SILVERMAN, 2006). ATCQ é uma técnica de diagnóstico por imagem com alta sensibilidade, aplicabilidade e precisão no diagnóstico de perda de massa óssea, assim como para a identificação de fraturas vertebrais (GRAMPP et al., 1996). Vários estudos realizados em humanos e animais verificaram que a densidade mineral óssea (DMO) obtida por meio de exames tomográficos é precisa (CHALMERS et al., 2006).

A análise quantitativa da DMO por TC permitiu a determinação precisa da radiodensidade do osso trabecular das vértebras dorsais avaliadas do grupo experimental (Tabela 1) e, conforme também demonstrado nessa tabela, não se observaram alterações significativas entre os valores de radiodensidade das três vértebras dorsais avaliadas nos animais do grupo experimental. Esta uniformidade nos resultados demonstra que qualquer uma destas regiões pode ser utilizada para estimar a DMO e sugerir desmineralização óssea sistêmica. Esta uniformidade também é citada em humanos, entretanto as primeiras vértebras lombares são preferencialmente utilizadas em exames de rotina nos pacientes para melhor padronização da técnica (GRAMPP et al., 1996). 
Tabela 1 - Valores individuais, médias e desvio padrão da radiodensidade do corpo vertebral da segunda (ROI 1), terceira (ROI 2) e quarta vértebra dorsal (ROI 3) em unidades Hounsfield (HU) de cinco tartarugas verdes juvenis após exame de tomografia computadorizada helicoidal.

\begin{tabular}{llll}
\hline Tartaruga marinha & ROI 1 & ROI 2 & ROI 3 \\
\hline 1 & $262,5 \mathrm{HU}$ & $275,5 \mathrm{HU}$ & $303 \mathrm{HU}$ \\
2 & $382,8 \mathrm{HU}$ & $287,1 \mathrm{HU}$ & $291,5 \mathrm{HU}$ \\
3 & $260,2 \mathrm{HU}$ & $303,7 \mathrm{HU}$ & $264,9 \mathrm{HU}$ \\
4 & $300,3 \mathrm{HU}$ & $248,1 \mathrm{HU}$ & $261,8 \mathrm{HU}$ \\
5 & $339,0 \mathrm{HU}$ & $248,1 \mathrm{HU}$ & $274,6 \pm 31,5 \mathrm{HU}^{\mathrm{a}}$ \\
Média \pm DP & $308,9 \pm 52,4 \mathrm{HU}^{\mathrm{a}}$ & $284,7 \pm 24,4 \mathrm{HU}^{\mathrm{a}}$ & \\
\hline
\end{tabular}

ROI - região de interesse; HU - unidades Hounsfield; DP - desvio padrão. Médias seguidas de letras que se repetem na mesma linha não diferem estatisticamente em nível de 5\% de significância pelo teste T de student.

Estudos em humanos demonstraram que o osso trabecular e a borda cortical do corpo vertebral são mais susceptíveis à desmineralização do que osso cortical dos ossos longos. As fraturas também ocorrem com maior frequência em locais com maior quantidade de osso trabecular, como os corpos vertebrais, fêmur proximal e rádio distal (GRAMPP et al., 1996; SCHÄCKE et al., 2002). Estudos experimentais com animais também utilizaram com sucesso a TC para avaliação da DMO da coluna lombar em cães, ovelhas e minipigs (QUARLES, 1992; COSTA et al., 2010). Este fato ressalta a importância das medidas de radiodensidade do osso trabecular das vértebras dorsais para o estabelecimento precoce de um diagnóstico de desmineralização óssea em tartarugas marinhas.

Conforme demonstrado nas tabelas 2 e 3 , os ossos pleurais e o osso neural apresentam maior radiodensidade quando comparados com o corpo vertebral, entretanto aqueles estão mais sujeitos a fraturas de origem traumática. Segundo BARTEN (2006), em decorrência da fusão existente dos corpos vertebrais com o osso neural, as fraturas nessa área podem resultar em lesões medulares e sinais neurológicos graves e irreversíveis. De acordo com VALENTE et al. (2007), essas fraturas podem ocorrer pela ação da hélice de embarcações ou do impacto do casco, mordidas de tubarão e pela ação de pescadores. Dessa forma, um diagnóstico precoce de desmineralização óssea por técnicas densitométricas pode possibilitar a adoção de medidas de manejo e terapêuticas preventivas para a manutenção da resistência da carapaça contra processos traumáticos, diminuindo a possibilidade da ocorrência de fraturas patológicas nesses animais.

Utilizou-se, para contenção química, propofol na dose de $5 \mathrm{mg} \mathrm{kg}^{-1}$ após venopunção jugular, sendo este protocolo eficaz e permitindo uma rápida recuperação dos animais após o procedimento. É citada a utilização de doses entre 3 a $5 \mathrm{mg} \mathrm{kg}^{-1}$ para realização de sedação e indução anestésica em tartarugas marinhas (SCHUMACHER\&YELEN, 2006).VALENTEetal.(2007) utilizaram a associação de ketamina e diazepam por via intravenosa para a realização de exames de TC em tartarugas marinhas cabeçudas (Caretta caretta) juvenis, com sucesso, entretanto, os autores citam a

Tabela 2 - Valores individuais, médias e desvio padrão da radiodensidade do osso neural ao nível do corpo vertebral da segunda (ROI 1), terceira (ROI 2) e quarta vértebras dorsais (ROI 3) em unidades Hounsfield (HU) de cinco tartarugas verdes juvenis após exame de tomografia computadorizada helicoidal.

\begin{tabular}{llll}
\hline Tartaruga-marinha & ROI 1 & ROI 2 & ROI 3 \\
\hline 1 & $960,0 \mathrm{HU}$ & $1041,3 \mathrm{HU}$ & $1045,0 \mathrm{HU}$ \\
2 & $1155,8 \mathrm{HU}$ & $1319 \mathrm{HU}$ & $1140,3 \mathrm{HU}$ \\
3 & $1019,6 \mathrm{HU}$ & $1044,0 \mathrm{HU}$ & $1003,0 \mathrm{HU}$ \\
4 & $1124,4 \mathrm{HU}$ & $1103,8 \mathrm{HU}$ & $1122,0 \mathrm{HU}$ \\
5 & $1039,7 \mathrm{HU}$ & $1071,0 \mathrm{HU}$ & $1155 \mathrm{HU}$ \\
Média \pm DP & $1059,9 \pm 79,6 \mathrm{HU}^{\mathrm{a}}$ & $1115,8 \pm 115,35 \mathrm{HU}^{\mathrm{a}}$ & $1093,1 \pm 65,8 \mathrm{HU}^{\mathrm{a}}$ \\
\hline
\end{tabular}

ROI - região de interesse; HU - unidades Hounsfield; DP - desvio padrão. Médias seguidas de letras que se repetem na mesma linha não diferem estatisticamente em nível de $5 \%$ de significância pelo teste T de student.

Ciência Rural, v.42, n.8, ago, 2012. 
Tabela 3 - Valores individuais, médias e desvio padrão da radiodensidade do osso pleural ao nível do corpo vertebral da segunda (ROI 1), terceira (ROI 2) e quarta vértebras dorsais (ROI 3) em unidades Hounsfield (HU) de cinco tartarugas verdes juvenis após exame de tomografia computadorizada helicoidal.

\begin{tabular}{llll}
\hline Tartaruga-marinha & ROI 1 & ROI 2 & ROI 3 \\
\hline 1 & $1079,8 \mathrm{HU}$ & $1133,8 \mathrm{HU}$ & $1080,5 \mathrm{HU}$ \\
2 & $1192,7 \mathrm{HU}$ & $1239,2 \mathrm{HU}$ & $1196,7 \mathrm{HU}$ \\
3 & $879,3 \mathrm{HU}$ & $955,65 \mathrm{HU}$ & $1056,3 \mathrm{HU}$ \\
4 & $1149,3 \mathrm{HU}$ & $1101,7 \mathrm{HU}$ & $1023,5 \mathrm{HU}$ \\
5 & $1056,6 \mathrm{HU}$ & $1107,1 \mathrm{HU}$ & $1154,7 \mathrm{HU}$ \\
Média \pm DP & $1071,3 \pm 125,4 \mathrm{HU}^{\mathrm{a}}$ & $1107,5 \pm 104,0 \mathrm{HU}^{\mathrm{a}}$ & $1102,3 \pm 80,0 \mathrm{HU}^{\mathrm{a}}$ \\
\hline
\end{tabular}

ROI - região de interesse; HU - unidades Hounsfield; DP - desvio padrão. Médias seguidas de letras que se repetem na mesma linha não diferem estatisticamente em nível de $5 \%$ de significância pelo teste T de student.

possibilidade desses exames serem realizados sem contenção química, uma vez que os estudos de TC com aparelhos helicoidais não são invasivos e a aquisição das imagens é feita em poucos segundos.

\section{CONCLUSÃO}

Conclui-se que a TCQ permitiu o estabelecimento de valores médios de radiodensidade do osso trabecular das vértebras dorsais, osso pleural e osso neural de tartarugas marinhas, até então inéditos na literatura. Estudos complementares com maior número de animais e em diferentes espécies e faixas etárias devem ser realizados para propiciar maior aplicabilidade clínica e experimental da TCQ em tartarugas marinhas.

\section{COMITÊ DE ÉTICA E BIOSSEGURANÇA}

Protocolo número 23082.006298/2011-65.

\section{REFERÊNCIAS}

ABOU-MADI, N. et al. Diagnosis of skeletal injuries in chelonians using computed tomography. Journal of Zoo and Wildlife Medicine, v.35, p.226-231, 2004. Disponível em: <http://www.bioone.org/doi/abs/10.1638/03-024>. Acesso em: 27 ago. 2011. doi: 10.1638/03-024.

ADAMS, J.E. Quantitative computed tomography. European Journal of Radiology, v.71, p.415-424, 2009. Disponível em: <http://www.sciencedirect.com/science/article/pii/S0720048 X09004343>. Acesso em: 27 ago. 2011. doi: 10.1016/ j.ejrad.2009.04.074.

BARTEN, S.L. Shell demage. In: MADER, D.R. Reptile medicine and surgery. 2.ed. St. Louis: Elservier Saunders, 2006. p.893-899.

CHALMERS, H.J. et al. Assessment of bone mineral density of the femoral head in dogs with early osteoarthritis. American Journal of Veterinary Research. v.67, n.5, p.796-800, 2006.
Disponível em: <http://avmajournals.avma.org/doi/abs/10.2460/ ajvr.67.5.796>. Acesso em: 27 ago. 2011. doi:10.2460/ ajvr.67.5.796.

COSTA, L.A.V.S. Bone demineralization in the lumbar spine of dogs submitted to prednisone therapy. Journal of Veterinary Pharmacology and Therapeutics. v.33, n.6, p.583-586, 2010. Disponível em: <http://onlinelibrary.wiley.com/doi/10.1111/ j.13652885.2010.01174.x/ abstract>. Acesso em: 14 nov. 2010. doi: $10.1111 /$ j.1365-2885.2010.01174.x.

GAUDRON, C. et al. Imagerie medicale appliquée a l'anatomie clinique des che'loniens en consultation. In: INTERNATIONAL CONGRESS ON TESTUDO GENUS, 2001, France. Proceedings... Hyeres-France: Chelonii, 2001. V.3. p.161-179.

GEORGE, R.H. Health problems and diseases of sea turtles. In: LUTZ, P.L.; MUSICK, J.A. The biology of sea turtles. Boca Raton: CRC, 1996. V.1, p.362-386.

GRAMPP, S. et al. Quantitative CT assessment of the lumbar spine and radius in patients with osteoporosis. American Journal of Roentgenology, v.167, p.133-140, 1996.

GUMPENBERGER, M.; HENNINGER, W. The use of computed tomography in avian and reptile medicine. Seminars in Avian Exotic Pet Medicine. v.10, p.174-180, 2001. Disponível em: <http://www.sciencedirect.com/science/article/ pii/S1055937X01800116>. Acesso em: 27 ago. 2011. doi: 10.1053/saep.2001.24674.

LAUER, S.K. et al. Quantitative ultrasonography for assessment of bone mineral density in the canine radius and tibia. Veterinary Radiology \& Ultrasound, v.50, n.1, p.97-103, 2009.

LUTCAVAGE, M.E. et al. Human impacts on sea turtle survival. In: LUTZ P.L.; MUSICK, J.A. The biology of sea turtles. Boca Raton: CRC, 1996. V.1, p.387-410.

MADER, D.R. Metabolic bone diseases. In: MADER, D.R. Reptile medicine and surgery. 2.ed. St. Louis: Elsevier Saunders, 2006. p.841-851.

MARIRIC, M.; GLUCK, O. Densitometry in glucocorticoidinduced osteoporosis. Journal of Clinical Densitometry, v.7, n.4, p.359-363, 2004. Disponível em: <http:// www.clinicaldensitometry.com/article/S1094-6950(06)600176/abstract>. Acesso em: 27 ago. 2011. doi: 10.1385/ JCD:7:4:359. 
QUARLES, L. Prednisone induced osteopenia in beagles: variable effects mediated by differential suppression of bone formation. Journal of Physiology Endocrinology Metabolism, v.263, p.136-141, 1992.

SAEED, I. et al. Quantitative computed tomography reveals the effects of race and sex on bone size and trabecular and cortical bone density. Journal of Clinical Densitometry: Assessment of Skeletal Health, v.12, n.3, p.330-336, 2009.

SCHACKE, H. et al. Mechanisms involved in the side effects of glucocorticoids. Pharmacology \& Therapeutics, v.96, n.1, p.23-43, 2002. Disponível em: <http://www.sciencedirect.com/ science/article/pii/S0163725802002978>. Acesso em: 27 ago. 2011. doi: 10.1016/S0163-7258(02)00297-8.
SCHUMACHER, J.; YELEN, T. Anesthesia and analgesia. In: MADER, D.R. Reptile Medicine and Surgery. 2.ed. St. Louis: Elsevier Saunders, 2006. p.442-452.

SILVERMAN, S. Diagnostic imaging. In: MADER, D.R. Reptile medicine and surgery. 2.ed. St. Louis: Elsevier Saunders, 2006. p.471-489.

VALENTE, A.L.S et al. Computed tomography of the vertebral column and coelomic structures in the normal loggerhead sea turtle (Caretta caretta). Veterinary Journal, v.174, p.362370, 2007. Disponível em: <http://www.sciencedirect.com/ science/article/pii/S1090023306001924>. Acesso em: 29 ago. 2011. doi: 10.1016/j.tvjl.2006.08.018. 